\title{
Activation of peroxymonosulfate by natural molybdenite for the degradation of atrazine
}

\author{
Leiduo Lai ${ }^{1, *}$ and Bo Lai ${ }^{1}$ \\ ${ }^{1}$ Department of Environmental Science and Engineering, School of Architecture and Environment, Sichuan University, Chengdu 610065, \\ China
}

\begin{abstract}
In this work, molybdenite was used as a catalyst to activate peroxymonosulfate (PMS) for atrazine (ATZ) degradation. First, the effects of molybdenite dosage (0.05-0.30 g/L), PMS dosage (0.02$0.15 \mathrm{mM})$ and the initial $\mathrm{pH}(3.0-11.0)$ in the aqueous solution on the ATZ degradation were investigated in the molybdenite/PMS system. The maximum degradation efficiency of ATZ (95.0\%) after 10 min treatment was obtained at the molybdenite dosage of $0.2 \mathrm{~g} / \mathrm{L}$, PMS dosage of $0.08 \mathrm{mM}$ and initial $\mathrm{pH}$ of 6.4 . The control experiments suggested that molybdenite, which activate PMS had better performance than commercial molybdenum disulfide. It was found that the degradation efficiency of ATZ under the optimal parameters was only $41.2 \%$ in the commercial molybdenum disulfide/PMS system. The free radicals in the molybdenite/PMS system were studied by quenching experiment. The results showed that both $\mathrm{SO}_{4}{ }^{-}$and $\mathrm{HO}^{\circ}$ were existed in the molybdenite/PMS system. In general, this study provided a natural catalyst for PMS activation and would urge us re-evaluate the capacity of natural materials as catalysts.
\end{abstract}

\section{Introduction}

Since the atrazine (ATZ), a kind of typical chlorinecontaining organic herbicide, has potential threat to environment and the human beings, its degradation in the aqueous solution has attracted extensive attention in recent years[1, 2]. The U.S. Environmental Protection Agency (U.S. EPA) classified the ATZ as a kind of environmental endocrine disruptors (EEDs) and persistent organic pollutants (POPs) [3-5]. In other words, ATZ has a long half-life and degradation-resistant nature. The conventional sewage disposal process can no longer meet the processing requirements.

Based on advanced oxidation processes (AOPs), quantities of processing technics have been studies to degrade ATZ, such as ozonation, photocatalysis, activation of hydrogen peroxide, persulfate (PS) and peroxymonosulfate (PMS) [6-11]. Among these treatment processes, activations of PS and PMS are more excellent than others. The PS or PMS activation method could generate sulfate radical $\left(\mathrm{SO}_{4}^{-{ }^{-}}\right)$, which has a longer lifetime and a broader $\mathrm{pH}$ application range than hydroxyl radical (HO')[12]. However, PS and PMS can hardly degrade pollutants without the activators due to the lack of the powerful reactive oxygen species (ROS, like $\mathrm{SO}_{4}{ }^{*-}, \mathrm{HO}^{\circ}$, superoxide radical $\left(\mathrm{O}_{2}{ }^{*}\right)$, etc. $)$ [13]. Besides, compared with PS, PMS has a asymmetric structure, indicating PMS could be easier activated by activators to produce ROS[14].

Plentiful materials, including transition metal materials ( $\mathrm{Fe}, \mathrm{Cu}, \mathrm{Ag}$, Co based catalysts) and carbonbased materials[15-18], were used as PMS activators.
Recently, researchers pointed out that some metal sulfides could also serve as activators for PMS activation. For example, Chen et al. found that molybdenum disulfide $\left(\mathrm{MoS}_{2}\right)$ could effectively activate PMS to degrade bisphenol $\mathrm{A}[19]$. In other words, low-valence molybdenum might have potential effect on PMS activation. Based on this conjecture, molybdenite, a cheap substitution of $\mathrm{MoS}_{2}$, was used as a PMS activator in this study.

In this work, ATZ was used as a model contaminant to investigate the property of molybdenite for PMS activation. The main objectives of this work are to: (1) study the effects of the key parameters (molybdenite dosage, PMS dosage and the initial $\mathrm{pH}$ ) on the degradation of ATZ, (2) investigate the main ROS in the molybdenite/PMS system and (3) confirm the superiority of molybdenite for PMS activation by control experiments.

\section{Materials and methods}

\subsection{Reagents}

PMS and ATZ ( $\geq 97 \%$ ) were purchased from Shanghai Anpel Laboratory Technologies Inc. The tert butyl alcohol (TBA), ethyl alcohol (EtOH) and sodium thiosulfate $\left(\mathrm{Na}_{2} \mathrm{~S}_{2} \mathrm{O}_{3}\right)$ were bought from Chengdu Kelong chemical reagent factory. The molybdenite was purchased from Haoyu stone crafts firm. Other chemicals used in this work were of analytical grade. Deionized water was used throughout the whole experiment process.

* Corresponding author: laileiduo@163.com 


\subsection{Experiment procedure}

In this work, ATZ stock solution $(5 \mathrm{mg} / \mathrm{L})$ was prepared in advance via dissolution in the deionized water. At each run, $150 \mathrm{~mL}$ prepared ATZ stock solution was added into the $250 \mathrm{~mL}$ beaker. To initiate the reaction, a known amount of PMS and molybdenite powder were added into beaker simultaneously. The reactions were carried out in the water bath, which equipped with a mechanical stir bar at $300 \mathrm{rpm}$. The reaction temperature in the water bath was controlled at $30 \pm 1^{\circ} \mathrm{C}$. The degradation efficiency of ATZ was measured at predetermined times by high performance liquid chromatography (HPLC, Agilent 1260 , USA). The sample at predetermined times was withdrawn with syringe and immediately filtered through a PTFE syringe filter disc $(0.45 \mu \mathrm{m})$. For each sample, excess $\mathrm{Na}_{2} \mathrm{~S}_{2} \mathrm{O}_{3}$ was used as the scavenger to quench ROS. All data shown in this work were averaged values of 3 replicate samples and the error bars in the figures represented standard deviation of the means.

\subsection{Analytical method}

The concentration changes of ATZ during the reaction process were detected by HPLC, which equipped with the Eclipse XDB C-18 $(5 \mu \mathrm{m}, 4.6 \times 250 \mathrm{~mm})$ column. The mobile phase was ultrapure water (A) and methanol (B), and the mobile phase ratio of A: B was 1:4 with a flow rate of $1.0 \mathrm{~mL} / \mathrm{min}$. In addition, the test wavelength was set at $\lambda=225 \mathrm{~nm}$. The characteristics of molybdenite were analyzed by X-ray powder diffraction (XRD). The effects of key parameters (i.e., molybdenite powder dosage $(0.05-0.30 \mathrm{~g} / \mathrm{L})$, PMS dosage $(0.02-0.15 \mathrm{mM})$ and initial $\mathrm{pH}(3.0-11.0))$ on the ATZ degradation were investigated systematically. The ROS in the molybdenite/PMS system were measured by quenching experiments. Moreover, to confirm the superiority of molybdenite for PMS activation, five control experiments (molybdenite alone, PMS alone, $\mathrm{MoS}_{2} / \mathrm{PMS}, \mathrm{MoS}_{2}$ alone and molybdenite/PMS systems) were conducted in this work.

\section{Results and discussion}

\subsection{Characteristics of molybdenite}

The XRD patterns of the fresh and the used molybdenite was shown in Figure 1. It is clear that the diffraction patterns of both samples well correspond to the JCPDS 37-1492 of $\mathrm{MoS}_{2}$. In other words, the active species in the molybdenite were $\mathrm{MoS}_{2}$. Compared with the fresh molybdenite, there was no new peaks in the used molybdenite, indicating the crystalline of the molybdenite was not changed after use.

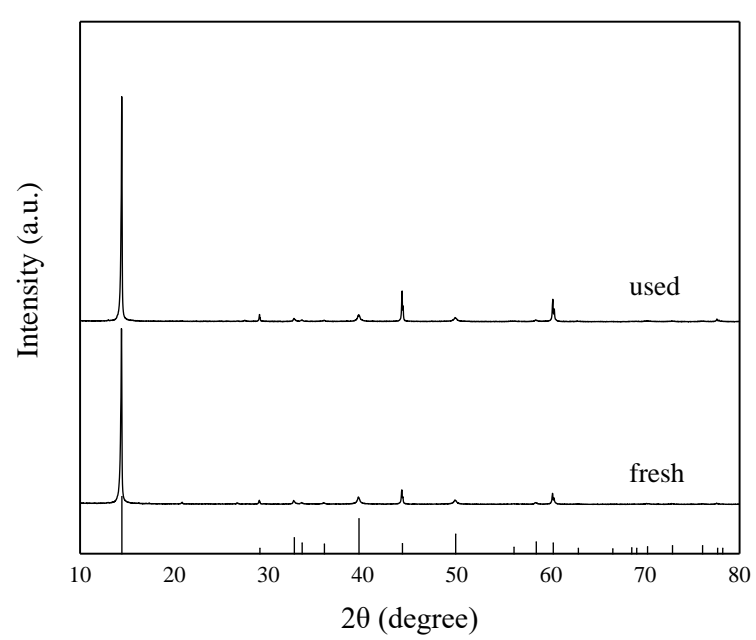

Fig. 1. XRD patterns of the fresh and the used molybdenite.

\subsection{Parameters optimization}

\subsubsection{Effect of molybdenite dosage on the degradation of $A T Z$}

For catalytic reaction, the catalyst dosage directly influences the amount of active sites[20]. In this study, the effect of the molybdenite dosage $(0.05-0.30 \mathrm{~g} / \mathrm{L})$ on the ATZ degradation in the molybdenite/PMS system was investigated systematically. Figure 2 suggests the degradation efficiency of ATZ increased with the increased dosage of molybdenite. Specifically, the degradation efficiency of ATZ increased from $17.6 \%$ to $97.8 \%$ when molybdenite dosage increased from 0.05 to $0.30 \mathrm{~g} / \mathrm{L}$, indicating the active sites for PMS activation increased with the molybdenite dosage. The degradation rate of ATZ increased slight as the molybdenite dosage increased from 0.20 to $0.30 \mathrm{~g} / \mathrm{L}$. The result manifested that active sites for $0.1 \mathrm{mM}$ PMS activation was enough when the dosage of molybdenite was $0.20 \mathrm{~g} / \mathrm{L}$. Therefore, the molybdenite dosage of $0.20 \mathrm{~g} / \mathrm{L}$ was selected in the following experiments.

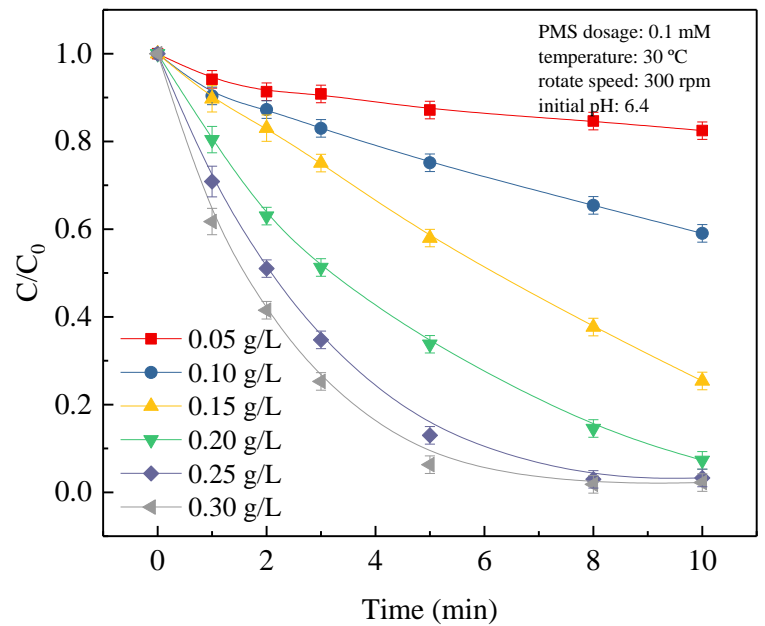

Fig. 2. Effect of molybdenite dosage $(0.05-0.30 \mathrm{~g} / \mathrm{L})$ on the degradation of ATZ. 


\subsubsection{Effect of PMS dosage on the degradation of ATZ}

In molybdenite/PMS system, the PMS was the precursor of the ROS[13]. Thus, the amount of PMS can significantly influence the amount of ROS. Figure 3 shows the ATZ degradation efficiency at different dosage of PMS (0.02-0.15 mM). The degradation efficiency of ATZ increased from $33.8 \%$ to $95.0 \%$ when PMS dosage increased from 0.02 to $0.08 \mathrm{mM}$. In other words, the more amount of PMS system, the more ROS generated in the molybdenite/PMS. However, the degradation efficiency of ATZ decreased from $95.0 \%$ to $81.6 \%$ when PMS dosage continued to increase $(0.08-0.15 \mathrm{mM})$. The phenomenon could be explained as the quenching effect of the ROS (Eq.(1) and (2)) [21]. According to the degradation efficiency of ATZ, $0.08 \mathrm{mM}$ PMS was selected as the optimal dosage in the following experiments.

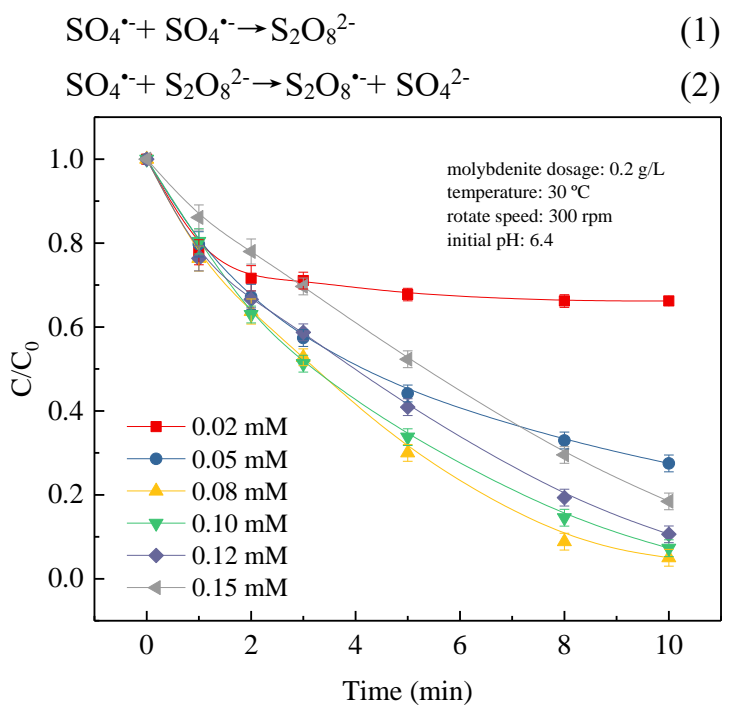

Fig. 3. Effect of PMS dosage $(0.02-0.15 \mathrm{mM})$ on the degradation of ATZ.

\subsubsection{Effect of initial $\mathrm{pH}$ on the degradation of ATZ}

The $\mathrm{pH}$ value in the solution could influence the existence form of contaminants, and then to affect the contaminants degradation efficiency [22]. In this work, the effect of different initial $\mathrm{pH}$ (3.0-11.0) on the degradation of ATZ was investigated. Figure 4 shows that ATZ could be effectively degraded at the initial $\mathrm{pH}$ of 3.0-6.4. Specifically, the ATZ degradation efficiency $(95.0 \%)$ was almost unchanged when the initial $\mathrm{pH}$ varied from 3.0 to 6.4. However, the degradation efficiency of ATZ declined to $80.0 \%$ and $9.1 \%$ when the initial $\mathrm{pH}$ increased to 9.0 and 11.0 , respectively. In other words, acidic conditions facilitated the ATZ degradation, while alkaline conditions inhibited the ATZ degradation. According to literatures, the pKa of ATZ was 1.68[23], therefore, ATZ obtained a negative charge in the experiment conditions. Besides, $\mathrm{HSO}_{5}^{-}$was the main active species when $\mathrm{pH}$ varied from 3.0 to 9.0 , while $\mathrm{SO}_{5}{ }^{2-}$ played an important role when $\mathrm{pH}$ at $11.0[24,25]$. $\mathrm{SO}_{5}{ }^{2-}$ has less catalytic activity than $\mathrm{HSO}_{5}$, indicating ATZ degradation efficiency decreased at $\mathrm{pH}$ of 11.0. The
$\mathrm{pH}$ value of ATZ aqueous solution without adjusting was 6.4, and the ATZ degradation efficiency at this $\mathrm{pH}$ was satisfactory. Therefore, the initial $\mathrm{pH}$ of 6.4 was selected as the optimal condition in the following experiments.

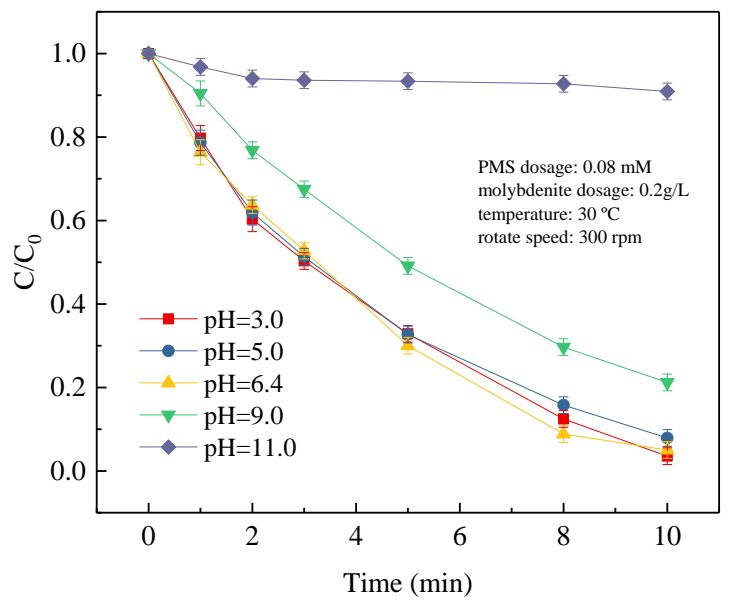

Fig. 4. Effect of initial $\mathrm{pH}(3.0-11.0)$ on the degradation of ATZ.

\subsection{Superiority of molybdenite}

To prove the superiority of molybdenite, ATZ was degraded in five different systems (i.e., molybdenite/PMS, molybdenite alone, PMS alone, $\mathrm{MoS}_{2} / \mathrm{PMS}$ and $\mathrm{MoS}_{2}$ alone systems). Figure 5 suggests that PMS without catalysts cannot degrade ATZ. Besides, both molybdenite and $\mathrm{MoS}_{2}$ had absorption effects on ATZ. After 10 min reaction, $6.9 \%$ and $8.8 \% \mathrm{ATZ}$ were absorbed in $\mathrm{MoS}_{2}$ alone and molybdenite alone systems. After adding catalysts in the PMS-activated processes, i.e., molybdenite and $\mathrm{MoS}_{2}$, ATZ degradation efficiencies were enhance significantly. However, the ATZ degradation efficiency in the molybdenite/PMS system (95.0\%) was much higher than that in the $\mathrm{MoS}_{2} / \mathrm{PMS}$ system $(41.2 \%)$. All the results manifested that catalytic activity of molybdenite was excellent for PMS activation.

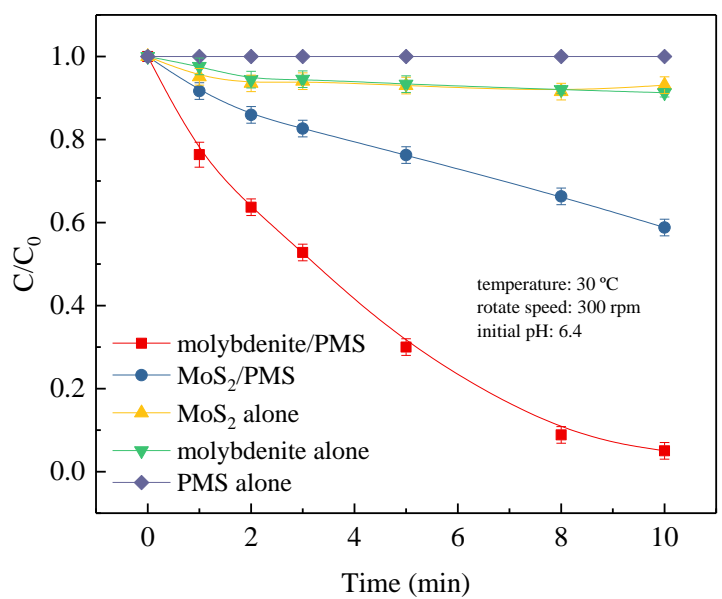

Fig. 5. The degradation efficiency of ATZ in different systems (molybdenite $=0.2 \mathrm{~g} / \mathrm{L}, \mathrm{MoS} 2=0.2 \mathrm{~g} / \mathrm{L}$ and $\mathrm{PMS}=0.08 \mathrm{mM}$ ).

\subsection{Quenching experiments}


ROS, like $\mathrm{SO}_{4}{ }^{--}$and $\mathrm{HO}^{\circ}$, would generate in the PMSactivated process[26]. To identify the dominant ROS involved in molybdenite/PMS system, quenching experiments were carried out by adding different scavengers. TBA was used as a $\mathrm{HO}^{*}$ scavenger $\left(k_{\mathrm{TBA}+}\right.$ $\left.\mathrm{HO}^{\circ}=(3.8-7.6) \times 10^{8} \mathrm{M}^{-1} \mathrm{~s}^{-1}, k_{\mathrm{TBA}}+\mathrm{SO}^{--}=(4-9.1) \times 10^{5} \mathrm{M}^{-1} \mathrm{~s}^{-1}\right)$ [27], while EtOH was used as a both $\mathrm{HO}^{-}$and $\mathrm{SO}_{4}^{-}$ scavenger $\left(k_{\mathrm{EtOH}}+\mathrm{SO} 4^{--}=3.5 \times 10^{7} \mathrm{M}^{-1} \mathrm{~s}^{-1}, k_{\mathrm{EtOH}}+\mathrm{HO}=9.1 \times 10^{6}\right.$ $\mathrm{M}^{-1} \mathrm{~s}^{-1}$ ) [28]. The data (Figure 6) shows that the ATZ degradation efficiency decreased from $95.0 \%$ to $18.8 \%$ and $9.0 \%$ with $50 \mathrm{mM}$ and $100 \mathrm{mM}$ EtOH added into molybdenite/PMS system. With addition of $50 \mathrm{mM}$ and100 $\mathrm{mM}$ TBA, the ATZ degradation efficiency decreased from $95.0 \%$ to $60.8 \%$ and $57.8 \%$, respectively. The results suggest that both $\mathrm{HO}^{*}$ and $\mathrm{SO}_{4}{ }^{-}$were formed, and $\mathrm{SO}_{4}{ }^{-}$was the dominant $\mathrm{ROS}$ responsible for ATZ degradation in the molybdenite/PMS system.

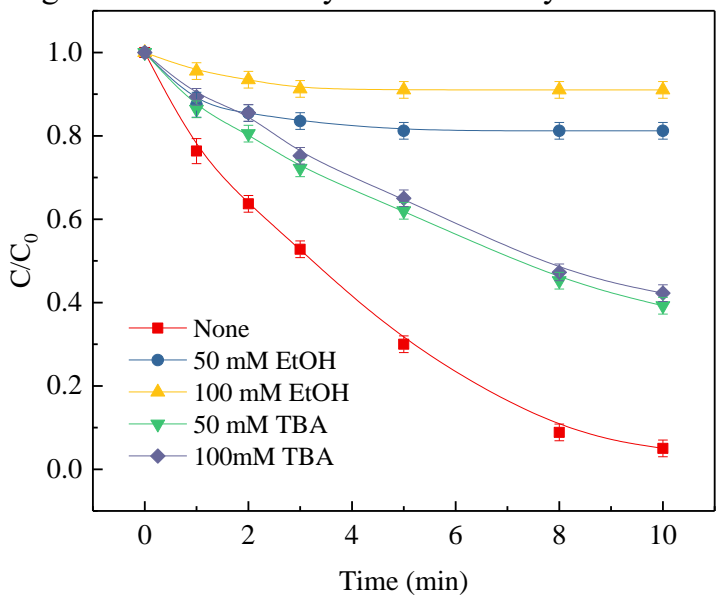

Fig. 6. Effects of TBA and EtOH on the ATZ degradation in the molybdenite/PMS system.

\section{4 conclusions}

This study provided a natural ore as an effective catalyst to activate PMS. Firstly, we investigated the effects of key parameters of molybdenite/PMS system on the ATZ degradation. Under experimental conditions (molybdenite dosage $=0.2 \mathrm{~g} / \mathrm{L}$, PMS dosage $=0.08 \mathrm{mM}$ and intial $\mathrm{pH}=6.4$ ), the degradation efficiency of ATZ could achieve $95.0 \%$. Compared with molybdenite alone, PMS alone, $\mathrm{MoS}_{2} / \mathrm{PMS}$ and $\mathrm{MoS}_{2}$ alone systems, molybdenite showed an excellent catalytic activity for PMS activation. Besides, the quenching tests manifested that both $\mathrm{HO}^{*}$ and $\mathrm{SO}_{4}{ }^{-}$existed in the molybdenite/PMS system to enhance the degradation of ATZ. In general, all the results suggested natural molybdenite could be used as a low-cost and effective catalyst in the environmental field.

The authors would like to acknowledge the financial support from National Natural Science Foundation of China (No.51878423).

\section{References}

1. A.M. Schmidt, N. Sengupta, C.A. Saski, R.E. Noorai, W.S. Baldwin, Chemosphere 189 (2017) 699-708.
2. F. Yang, W. Zhang, J. Li, S. Wang, Y. Tao, Y. Wang, Y. Zhang, Chemosphere 189 (2017) 507-516.

3. C. Chen, S. Yang, Y. Guo, C. Sun, C. Gu, B. Xu, J Hazard Mater 172 (2009) 675-684.

4. I. de la Casa-Resino, A. Valdehita, F. Soler, J.M. Navas, M. Perez-Lopez, Comp Biochem Physiol C Toxicol Pharmacol 156 (2012) 159-165.

5. X. Kong, J. Jiang, J. Ma, Y. Yang, W. Liu, Y. Liu, Water Res 90 (2016) 15-23.

6. L. Lai, J. Yan, J. Li, B. Lai, Chemical Engineering Journal 343 (2018) 676-688.

7. E. Illes, E. Szabo, E. Takacs, L. Wojnarovits, A. Dombi, K. Gajda-Schrantz, Sci Total Environ 472 (2014) 178-184.

8. F. Chen, H. Huang, Y. Zhang, T. Zhang, Chinese Chemical Letters 28 (2017) 2244-2250.

9. M. Feng, V.K. Sharma, Chemical Engineering Journal (2018).

10. S. Zhang, S. Gitungo, L. Axe, J.E. Dyksen, R.F. Raczko, Water Res 105 (2016) 85-96.

11. G. Ayoub, A. Ghauch, Chemical Engineering Journal 256 (2014) 280-292.

12. P. Hu, M. Long, Applied Catalysis B Environmental 181 (2016) 103-117.

13. L. Lai, J. Yan, J. Li, B. Lai, Chemical Engineering Journal 343 (2018) 676-688.

14. X. Chen, W. Wang, H. Xiao, C. Hong, F. Zhu, Y. Yao, Z. Xue, Chemical Engineering Journal 193 (2012) 290-295.

15. H. Li, C. Shan, W. Li, B. Pan, Water Res 147 (2018) 233-241.

16. G.X. Huang, C.Y. Wang, C.W. Yang, P.C. Guo, H.Q. Yu, Environ Sci Technol 51 (2017) 12611-12618.

17. G.P. Anipsitakis, D.D. Dionysiou, Environmental Science \& Technology 38 (2004) 3705.

18. X. Duan, Z. Ao, L. Zhou, H. Sun, G. Wang, S. Wang, Applied Catalysis B Environmental 188 (2016) 98105.

19. Y. Chen, G. Zhang, H. Liu, J. Qu, Angew Chem Int Ed Engl 58 (2019) 8134-8138.

20. L. Lai, H. Zhou, B. Lai, Chemical Engineering Journal 349 (2018) 633-645.

21. J.M. Monteagudo, A. Durán, R. González, A.J. Expósito, Applied Catalysis B: Environmental 176177 (2015) 120-129.

22. Y. Lei, C.S. Chen, Y.J. Tu, Y.H. Huang, H. Zhang, Environ Sci Technol 49 (2015) 6838-6845.

23. S. Wu, H. He, X. Li, C. Yang, G. Zeng, B. Wu, S. He, L. Lu, Chemical Engineering Journal 341 (2018) 126-136.

24. T. Zhang, H. Zhu, J.P. Croue, Environ Sci Technol 47 (2013) 2784-2791.

25. F. Ghanbari, M. Moradi, Chemical Engineering Journal 310 (2017) 41-62. 
26. P. Hu, M. Long, Applied Catalysis B: Environmental 181 (2016) 103-117.

27. Y. Xu, J. Ai, H. Zhang, Journal of hazardous materials 309 (2016) 87-96.
28. E. Hayon, A. Treinin, J. Wilf, Electronic spectra, Journal of the American Chemical Society 94 (1972) 47-57. 